\title{
Comparación de métodos de optimización para un experimento con múltiples variables de respuesta
}

\section{Optimization methods comparison for an experiment with multiple response variables}

Judith Mendoza-Gallardo1, Luis Alberto Rodríguez-Picón1, Luis Carlos Méndez-González1

1 Universidad Autónoma de Ciudad Juárez

\section{RESUMEN}

Dentro del proceso de experimentación estadística existen ciertas variables que en conjunto determinan las condiciones ideales de múltiples variables de respuesta. Un reto importante dentro de la optimización de modelos creados a partir de experimentos recae precisamente en las situaciones en que la calidad de un producto es función de múltiples variables de respuesta y dadas estas condiciones resulta necesario establecer estrategias precisas que permitan optimizar todas las respuestas a la vez. En este trabajo se experimentó con tres métodos de optimización con el propósito de encontrar el mejor de ellos y la estrategia capaz de optimizar las funciones que consisten en más de una característica crítica. El objetivo del artículo es encontrar la estrategia capaz de arrojar las condiciones óptimas de dos variables de respuesta, condicionadas por restricciones iniciales. Los resultados de optimización obtenidos a través de los métodos considerados fueron similares en cuanto al logro de los objetivos de optimización planteados.

PALABRAS CLAVE: múltiples variables de respuesta; programación no lineal; optimización; experimento.

\section{ABSTRACT}

In the process of statistical experimentation there are certain variables that determine the ideal conditions for multiple response variables. An important challenge in the optimization of models created from experimental designs relies precisely in situations where the quality of a product is a function of multiples response variables; under these circumstances it is necessary to establish precise strategies that allow optimizing all the response variables as a whole. Within this article, three optimization methods are experimented with in order to find the best method and strategy able to optimize the functions, which consist of more than one critical characteristic. The objective of the article is to find the optimal strategy capable of delivering the optimal conditions of two response variables, conditioned by initial restrictions. The optimization results obtained through the methods considered were similar in terms of achieving the proposed optimization objectives.

KEYWORDS: multiple response variables; non-linear programming; optimization; experiment.

\section{Correspondencia:}

DESTINATARIO: Luis Alberto Rodríguez Picón INSTITUCIÓN: Universidad Autónoma de Ciudad Juárez DIRECCIÓN: Av. del Charro núm. 450 norte, col. Partido Romero, C. P. 32310, Ciudad Juárez, Chih., México CORREO ELECTRÓNICO: luis.picon@uacj.mx
Fecha de recepción: 22 de octubre de 2021. Fecha de aceptación: 10 de junio de 2021. Fecha de publicación: 19 de junio de 2021.
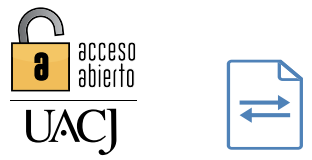


\section{INTRODUCCIÓN}

Desde los años 80 y hasta el día de hoy, el diseño de experimentos se ha fijado en un método de confianza, hasta convertirse en una herramienta fundamental para la resolución de problemas en muchos ámbitos de la vida industrial ${ }^{[1]}$.

Es común realizar pruebas en diferentes ámbitos prácticos, ya sea para la resolución de alguna inconformidad o para efectuar una comprobación de hipótesis, por ejemplo, hacer cambios en la composición de cierto producto, realizar modificaciones en la temperatura de cocción para algún platillo, optimizar el funcionamiento de un equipo para la fabricación de algún producto, etc.

Dentro del ramo industrial siempre ha existido la imperante necesidad de analizar en conjunto ciertos factores, contemplando siempre la probabilidad de que el efecto de un componente varíe según el nivel de los restantes, es decir, que exista una correlación. En la actualidad se reconoce al diseño de experimentos (DOE, por sus siglas en inglés), como una excelente herramienta capaz de lograr la optimización de los valores de respuesta mediante la combinación más eficaz de componentes [2].

Se reconoce este método como la manera más eficaz de realizar experimentaciones, pues básicamente identifica las pruebas necesarias para conseguir un resultado óptimo y confiable obtenido mediante un análisis, lo cual permitirá la resolución de ciertos problemas o la consecución de mejoras tangibles. En la práctica se presentan una variedad de situaciones y problemas que afectan el desarrollo de cierta actividad, por lo cual existen muchos diseños experimentales. Por ello, resulta imprescindible elegir el método adecuado para cada condición.

En pocas palabras, el diseño de experimentos consiste en planear y realizar un conjunto de pruebas con el objetivo de generar datos que, al ser analizados estadísticamente, proporcionan evidencias objetivas que permitan responder a las interrogantes planteadas por el experimentador sobre determinada situación [3].

Por lo general, solo se considera una variable de respuesta, lo que da origen a una optimización simple. Sin embargo, en muchos casos, la calidad de los procesos está representada por diversas características de interés.
En estos casos se consideran modelos de optimización para múltiples variables de respuesta y el objetivo es definir un conjunto de factores que proporcionen la mejor mediación simultánea de las múltiples variables de respuesta para alcanzar, de esta forma, la calidad global de un producto ${ }^{[4]}$.

La mayoría de los productos fabricados en la industria cuentan con una o más características de calidad que podrían considerarse de suma importancia para el consumidor. Por ejemplo, una pieza fabricada por el método de inyección de plástico se identifican las dimensiones críticas como características de calidad, en el caso de que se aplique un cambio en algún parámetro, verbigracia, una alteración en la presión de la línea que pudiese ocasionar una variación en el resultado de dichas características en proporciones diferentes.

Por otra parte, mediante la modificación en el diseño de la línea o los componentes es posible reducir la variación de ciertas dimensiones críticas. Por ende, es preferible tratar de generar cambios en el diseño del proceso, ya que, de esta manera, es probable disminuir la variación en una de las variables de respuesta sin afectar de manera negativa el resultado de otra ${ }^{[5]}$.

A menudo, tanto en la industria como en muchas áreas científicas, se obtienen datos sobre múltiples variables de respuesta para un nivel dado de un conjunto de variables controlables. Un experimento en el cual un número de variables respuestas se miden simultáneamente para cada nivel de un conjunto de variables controlables se denomina un experimento de múltiples variables de respuesta [6].

Las variables que puedan afectar las características de calidad individuales no deben experimentarse apartadas de los efectos que puedan tener en otras características de calidad importantes, es decir, deben considerarse conjuntamente los efectos de los cambios en las variables de diseño del proceso en todas las características de calidad importantes. Es necesario, entonces, encontrar métodos y estrategias que permitan optimizar los procesos de fabricación de productos que consisten en más de una característica crítica de calidad y/o por más de un parámetro de proceso crítico ${ }^{[5]}$.

Teóricamente, las técnicas de optimización con múltiples variables de respuesta consisten en seleccionar los niveles óptimos de factores, con el fin de optimizar todas 
las respuestas a la vez. Sin embargo, en la práctica esta situación puede resultar compleja, ya que es probable que se presente un conflicto producto de alguna interacción. En la literatura se han presentado aportaciones importantes en el área de optimización de múltiples respuestas. He et al. [7] propusieron una estrategia de optimización robusta basada en funciones de deseabilidad, de manera conjunta con el método de búsqueda simplex para encontrar las múltiples respuestas óptimas. Shamsudeen et al. ${ }^{[8]}$ y Jambhale et al. ${ }^{[9]}$ consideraron diferentes esquemas de experimentación para optimizar múltiples parámetros de un proceso de soldadura por fricción-agitación de punto. Kilic et al. ${ }^{[10]}$ asumieron la metodología de superficie de respuesta (MSR) y el análisis envolvente de datos para optimizar el ácido fítico y el volumen de pan simultáneamente. Awad et al. [11] tomaron en cuenta la MSR y un enfoque de optimización multiobjetivo para la optimización de mezclas de combustible y aceite de fusel con diferentes contenidos de agua. Mia ${ }^{[12]}$ y Achuthamenon et al. ${ }^{[13]}$ presentaron esquemas de optimización multiobjetivo para un proceso de fresado y aunque consideraron diferentes esquemas de experimentación, optimizaron a través de funciones de deseabilidad.

En este artículo se presenta una comparación de tres métodos de optimización encontrados en la literatura que son comúnmente utilizados cuando se estudian procesos con múltiples variables de respuesta. Se considera un modelo ajustado a partir de un experimento factorial, así como un conjunto de restricciones de interés para estudiar los resultados obtenidos del método de programación no lineal, funciones de deseabilidad y la superposición de gráficos de contorno.

El resto del artículo está organizado de la siguiente manera: en la sección II se presenta y describe el método considerado en el presente artículo; en la sección III se muestra el análisis estadístico del experimento bajo estudio con la finalidad de obtener los modelos ajustados, así como los resultados en cuanto a la comparación de los diferentes métodos analizados y, por último, en la sección IV se exponen las conclusiones.

\section{A. Métodos de optimización para experimentos con múltiples variables de respuesta}

Existen diferentes técnicas para la optimización de múltiples variables de respuesta, las cuales se presentan a continuación.

\section{Método gráfico}

Dentro de la programación lineal se manipulan solo dos factores $y$, por ende, resulta fácil trazar las superficies de respuesta, aunque este método resulta complejo al operar más de dos factores, puesto que las superficies no se pueden dibujar de una sola vez. En estos casos, resulta posible realizar el análisis tomando dos factores a la vez y fijando a los restantes ${ }^{[14]}$.

El método gráfico de optimización simultánea consiste en ajustar los modelos y sobreponer dos curvas de nivel de cada superficie sobre diferentes cortes bidimensionales de un cubo, de tal manera que la ubicación específica de un corte está dictada por el valor obtenido del tercer factor, esto para cada sección [15].

El punto óptimo general se representa como una esfera en algún lugar del cubo y en cada una de las hojas de corte se describe una circunferencia, cuyo centro intercepta a la circunferencia más grande. De esta manera es posible identificar el sitio óptimo ${ }^{[14]}$.

\section{Funciones de deseabilidad}

El método consiste en definir una función en el espacio de factores que mide la deseabilidad global (DG) del producto predicha en cada punto, transformando de esta manera el problema de optimización multivariado en univariado, y solo basta maximizar dicha deseabilidad global para obtener el punto óptimo ${ }^{[16]}$. En general, el método tiene la finalidad de encontrar las condiciones de operación de un conjunto de factores que provean las respuestas más deseables.

Para cada variable de respuesta $y_{i}(x)$, una función de deseabilidad individual asigna números entre 0 y 1 para los posibles valores de $y_{i}$, con $d_{i}\left(y_{i}\right)=0$, representando un valor completamente indeseado de $y_{i}$ y $d_{i}\left(y_{i}\right)=1$, valor de la respuesta completamente deseable. Una vez calculadas las $k$ deseabilidades individuales, se obtiene DG como la media geométrica ${ }^{[17]}$ :

$$
D G=\left\{d_{1}\left(y_{1}\right) \times d_{2}\left(y_{2}\right) \times \ldots \times d_{k}\left(y_{k}\right)\right\}^{1 / k}
$$

en donde $i=1,2, \ldots, k$ denota el número de variables de respuesta. En aplicaciones prácticas de este método se considera utilizar respuestas ajustadas $\hat{y}_{i}$. Las funciones de deseabilidad individual se pueden encontrar en la li- 
teratura dependiendo de la necesidad de minimizar o maximizar el conjunto de respuestas [18], [19].

El punto óptimo simultáneo es el punto

$$
X_{\mathrm{o}} 0=\left(X_{10}, X_{20}, \ldots, X_{p 0}\right)
$$

sobre el cual la función DG es máxima ${ }^{[10]}$.

\section{Programación no lineal}

Se considera programación no lineal al conjunto de métodos utilizados para optimizar una función objetivo, sujeta a una serie de restricciones en las que una o más de las variables incluidas es no lineal. Si la función objetivo o alguna de las restricciones llegan a ser no lineales y las variables solo consiguen tomar valores enteros no negativos, entonces se dice que el modelo matemático pertenecerá al campo de la programación no lineal entera ${ }^{[20]}$.

Los problemas con estas características surgen de forma inevitable en algunas aplicaciones de ingeniería, tales como diseño y control óptimo. Existen muchos tipos de problemas de programación no lineal dependiendo de las características de estas funciones, por lo que se han desarrollado distintos algoritmos para poder así resolver los distintos problemas. Para ciertos casos donde las funciones tienen formas sencillas, los problemas pueden llegar a resolverse de manera relativamente eficiente y en algunos otros, cuando se consideran modelos de mayor orden o con interacciones de un gran nivel, el proceso de optimización resulta complejo.

Cuando un problema de programación no lineal cuenta con solo una o dos variables, puede ser representada en forma gráfica. Si las funciones no son lineales, se trazan algunas curvas en lugar de rectas, por lo que la función objetivo y la región factible dejarán de mostrar el semblante que llevan en la programación lineal [21].

De manera general, este método de optimización tiene el siguiente planteamiento:

$$
\begin{gathered}
\text { mín } f(x) \\
\text { s. } a . \\
l_{h} \leq h(x) \leq U_{h} \\
l_{x} \leq x \leq U_{x}
\end{gathered}
$$

en donde, $f(x)$ representa la función objetivo, $x$ el conjunto de factores a optimizar y $h(x)$ un modelo formulado a partir de $x$.

\section{METODOLOGÍA}

En la Figura 1 se presenta un diagrama de flujo que refleja el método considerado para el estudio presentado en este artículo. A continuación, se describe de manera general cada paso.

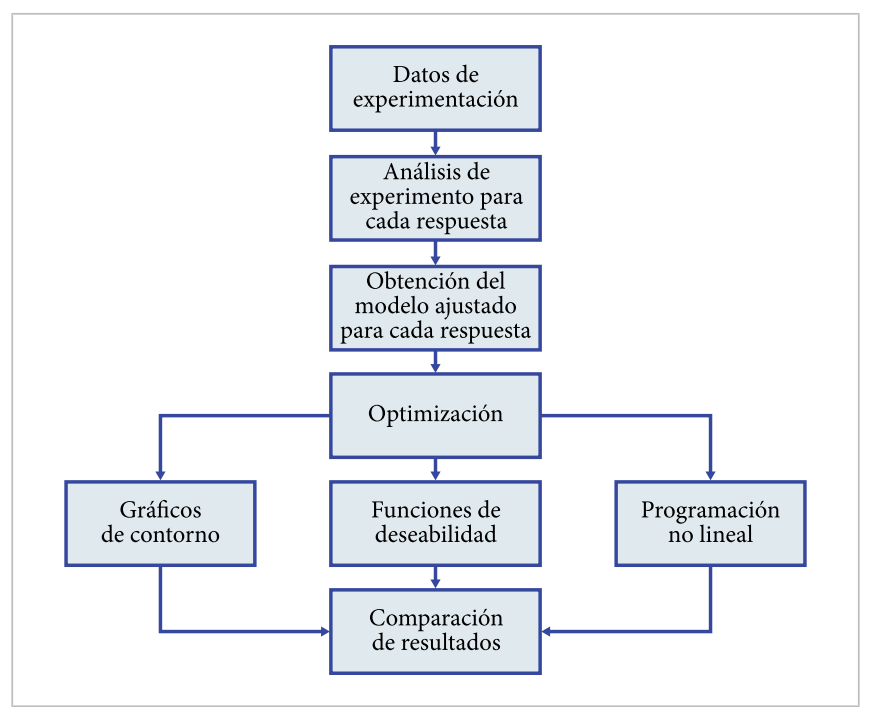

Figura 1. Método considerado en el presente estudio.

Datos de experimentación. De manera general, este paso consiste en llevar a cabo alguna estrategia de experimentación apropiada. La intención es disponer de un conjunto de datos obtenidos bajo condiciones controladas, con la finalidad de establecer un modelo apropiado que describa el proceso de interés. En este artículo se considera un experimento presentado en la literatura que considera múltiples variables de respuesta.

Análisis de experimento para cada respuesta. Este paso de método consiste en llevar a cabo un análisis estadístico del experimento para cada respuesta, que consiste en definir qué términos de este resultan significantes para cada respuesta, así como verificar el comportamiento de residuos con la finalidad de validar los modelos que se pretende ajustar.

Obtención del modelo ajustado para cada respuesta. En este paso se formulan los modelos a partir de los coeficientes de regresión ajustados para cada variable de respuesta. 
Optimización. En este paso se llevan a cabo las tres estrategias de optimización presentadas en la sección I. Para los métodos de gráficos de contorno y funciones de deseabilidad se usa el software Minitab ${ }^{\circledR}$, mientras que para el método de programación no lineal se considera el software estadístico R.

Comparación de resultados. Por último, se realiza una comparación de los resultados obtenidos y de igual manera se discuten los valores óptimos encontrados para cada respuesta.

\section{RESULTADOS Y DISCUSIÓN}

Se considera un experimento que consiste en tres variables controlables y en el que cada una de ellas es observada en dos niveles, los cuales se encuentran codificados de tal manera que -1 supone un nivel bajo mientras que +1 representa un nivel alto. En los niveles codificados, los valores actuales de las variables controlables son equivalentes a cero.

Como se muestra en la Tabla 1, para cada combinación propia de las tres variables controlables, son observadas cuatro réplicas de las dos variables de respuesta. Dada esta condición, para llevar a cabo el experimento se empleó un diseño de $2^{3}$ para las variables controlables con cuatro réplicas de la respuesta bivariada presente. Para este ejercicio en particular, el ruido experimental se manifiesta sencillamente por la variabilidad aleatoria, ya que no se cuenta con factores de ruido explícitos.

\section{A. Análisis del experimento para cada respuesta}

A continuación, se presenta el análisis de los experimentos para las dos respuestas, para lo cual se consideró Minitab ${ }^{\circledR}$.

La Figura 2 presenta el gráfico obtenido al realizar la prueba de normalidad de residuales para la primera respuesta $Y_{1}$, en donde se observa que no existe un comportamiento diferente al normal, puesto que con un nivel de significancia de 0.05 , se dictamina que no existe suficiente evidencia para rechazar la hipótesis nula que asume normalidad.

En la Figura 3 se muestra a la vez el análisis 4 en 1 para los residuos de la respuesta $Y_{1}$, donde rápidamente es posible detectar la independencia de residuales en el cua- dro inferior derecho, dado que no hay presencia de tendencias y que los residuales se varían alrededor del cero.

TABLA 1

DATOS DEL EXPERIMENTO [5]

\begin{tabular}{|c|c|c|c|c|c|c|}
\hline FILA & $X_{1}$ & $X_{2}$ & $X_{3}$ & RÉPLICA & $Y_{1}$ & $Y_{2}$ \\
\hline 1 & -1 & -1 & -1 & 1 & 109.895 & 67.6974 \\
\hline 2 & 1 & -1 & -1 & 2 & 100.192 & 67.0264 \\
\hline 3 & -1 & 1 & -1 & 3 & 106.078 & 72.9353 \\
\hline 4 & 1 & 1 & -1 & 4 & 104.120 & 72.9878 \\
\hline 5 & -1 & -1 & 1 & 5 & 113.515 & 68.2934 \\
\hline 6 & 1 & -1 & 1 & 6 & 98.735 & 67.0955 \\
\hline 7 & -1 & 1 & 1 & 7 & 103.145 & 71.6818 \\
\hline 8 & 1 & 1 & 1 & 8 & 104.454 & 76.9003 \\
\hline 9 & -1 & -1 & -1 & 1 & 109.759 & 67.2374 \\
\hline 10 & 1 & -1 & -1 & 2 & 99.634 & 66.1779 \\
\hline 11 & -1 & 1 & -1 & 3 & 105.642 & 72.8508 \\
\hline 12 & 1 & 1 & -1 & 4 & 104.802 & 74.2487 \\
\hline 13 & -1 & -1 & 1 & 5 & 111.121 & 68.4693 \\
\hline 14 & 1 & -1 & 1 & 6 & 99.357 & 63.6112 \\
\hline 15 & -1 & 1 & 1 & 7 & 106.959 & 76.2657 \\
\hline 16 & 1 & 1 & 1 & 8 & 105.029 & 77.0322 \\
\hline 17 & -1 & -1 & -1 & 1 & 110.704 & 67.9620 \\
\hline 18 & 1 & -1 & -1 & 2 & 100.269 & 66.5778 \\
\hline 19 & -1 & 1 & -1 & 3 & 105.670 & 72.5756 \\
\hline 20 & 1 & 1 & -1 & 4 & 104.203 & 73.9371 \\
\hline 21 & -1 & -1 & 1 & 5 & 112.854 & 68.9576 \\
\hline 22 & 1 & -1 & 1 & 6 & 102.842 & 68.9470 \\
\hline 23 & -1 & 1 & 1 & 7 & 107.620 & 77.4958 \\
\hline 24 & 1 & 1 & 1 & 8 & 99.786 & 67.9890 \\
\hline 25 & -1 & -1 & -1 & 1 & 109.773 & 66.9268 \\
\hline 26 & 1 & -1 & -1 & 2 & 100.600 & 67.9431 \\
\hline 27 & -1 & 1 & -1 & 3 & 105.393 & 72.3752 \\
\hline 28 & 1 & 1 & -1 & 4 & 104.335 & 73.2824 \\
\hline 29 & -1 & -1 & 1 & 5 & 106.666 & 64.7051 \\
\hline 30 & 1 & -1 & 1 & 6 & 94.235 & 62.4188 \\
\hline 31 & -1 & 1 & 1 & 7 & 103.440 & 76.3739 \\
\hline 32 & 1 & 1 & 1 & 8 & 104.923 & 75.7691 \\
\hline
\end{tabular}

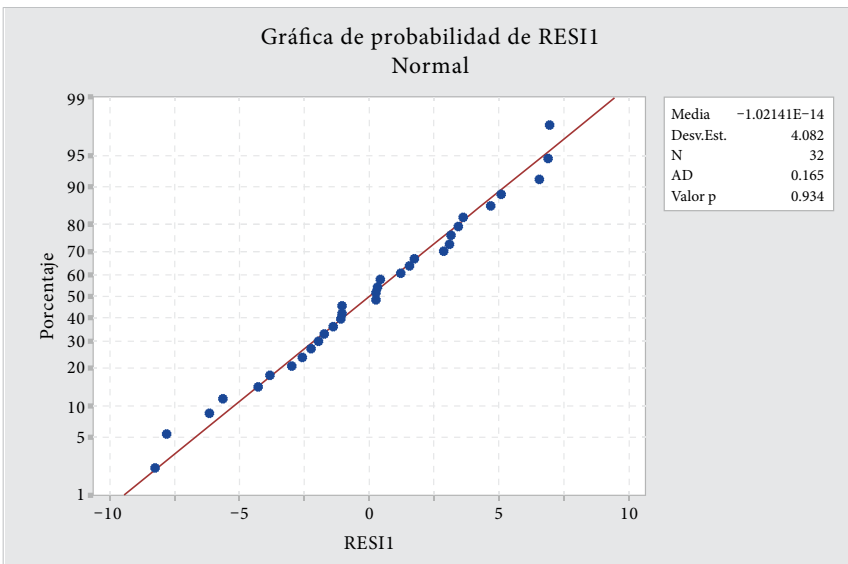

Figura 2. Gráfico de normalidad de residuales $Y_{1}$. 


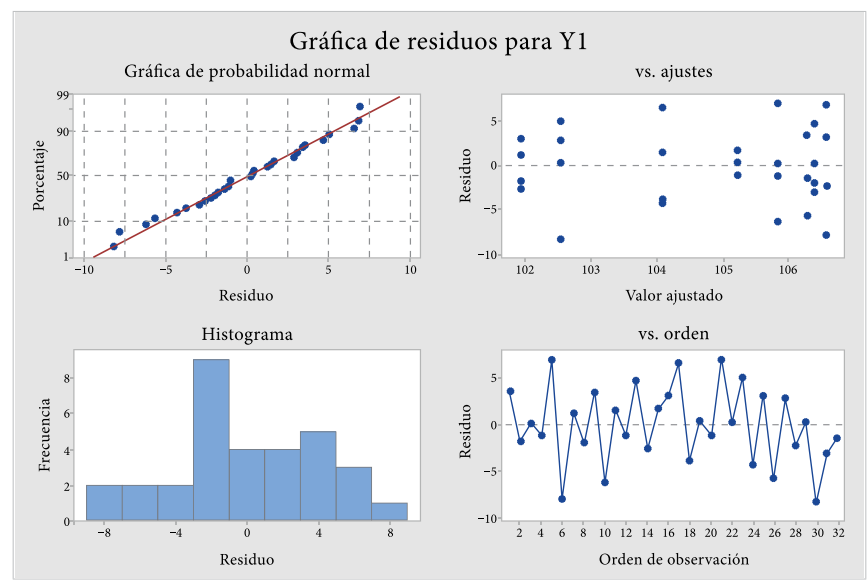

Figura 3. Independencia de residuales $Y_{1}$.

La Tabla 2 presenta los resultados del análisis de varianza (ANOVA) donde, dado un nivel de significancia de 0.05 , se puede notar que ninguno de los términos analizados es significante al encontrar que todos los correspondientes valores $\mathrm{p}$ son mayores que el nivel de significancia de 0.05 .

TABLA 2

ANOVA PARA $Y_{1}$

\begin{tabular}{|l|c|r|r|r|r|}
\hline \multicolumn{1}{|c|}{ FUENTE } & GL & \multicolumn{1}{c|}{$\begin{array}{c}\text { SC } \\
\text { AJUST. }\end{array}$} & $\begin{array}{c}\text { MC } \\
\text { AJUST. }\end{array}$ & $\begin{array}{c}\text { VALOR } \\
\mathrm{F}\end{array}$ & $\begin{array}{c}\text { VALOR } \\
\mathrm{p}\end{array}$ \\
\hline Modelo & 7 & 92.834 & 13.2620 & 0.62 & 0.737 \\
\hline Lineal & 3 & 79.977 & 26.6592 & 1.24 & 0.317 \\
\hline$X_{1}$ & 1 & 64.454 & 64.4538 & 3.00 & 0.096 \\
\hline$X_{2}$ & 1 & 0.367 & 0.3670 & 0.02 & 0.897 \\
\hline$X_{3}$ & 1 & 15.157 & 15.1566 & 0.70 & 0.410 \\
\hline Interacciones de 2 términos & 3 & 12.822 & 4.2740 & 0.20 & 0.896 \\
\hline$X_{1} \times X_{2}$ & 1 & 3.386 & 3.3859 & 0.16 & 0.695 \\
\hline$X_{1} \times X_{3}$ & 1 & 9.092 & 9.0919 & 0.42 & 0.522 \\
\hline$X_{2} \times X_{3}$ & 1 & 0.344 & 0.3442 & 0.02 & 0.900 \\
\hline Interacciones de 3 términos & 1 & 0.034 & 0.0344 & 0.00 & 0.968 \\
\hline$X_{1} \times X_{2} \times X_{3}$ & 1 & 0.034 & 0.0344 & 0.00 & 0.968 \\
\hline Error & 24 & 516.423 & 21.5176 & & \\
\hline Total & 31 & 609.256 & & & \\
\hline
\end{tabular}

La variable de respuesta $Y_{2}$ fue sometida al mismo análisis, en primera instancia, a una prueba de normalidad para residuos, donde con un nivel de significancia de 0.05 y dado un valor $\mathrm{p}$ de 0.532 no existe suficiente evidencia estadística para rechazar la hipótesis nula, la cual enuncia la normalidad de residuos (Figura 4).

Para la prueba de independencia, se interpretó el análisis 4 en 1 (Figura 5), donde se puede observar la falta de tendencia y la presencia de movimientos oscilantes alrededor del 0 , lo cual es una característica del supuesto de independencia.

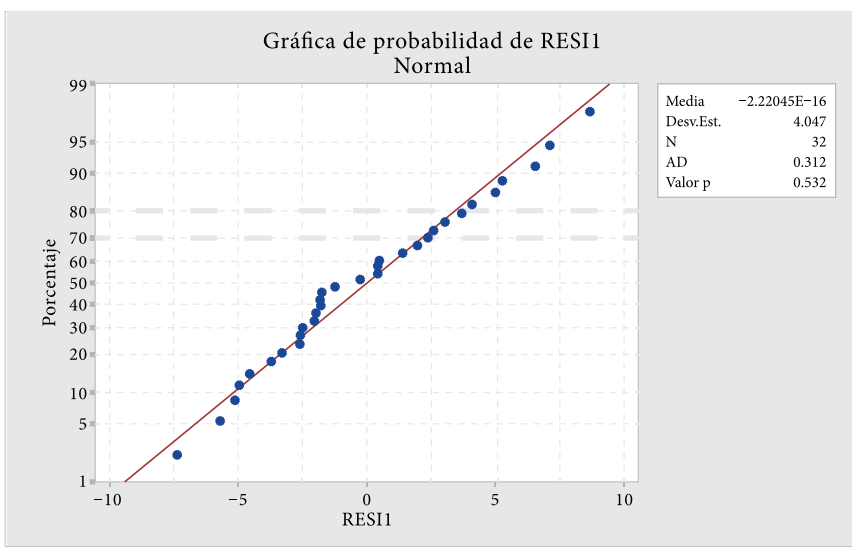

Figura 4. Gráfico de normalidad de residuales $Y_{2}$.

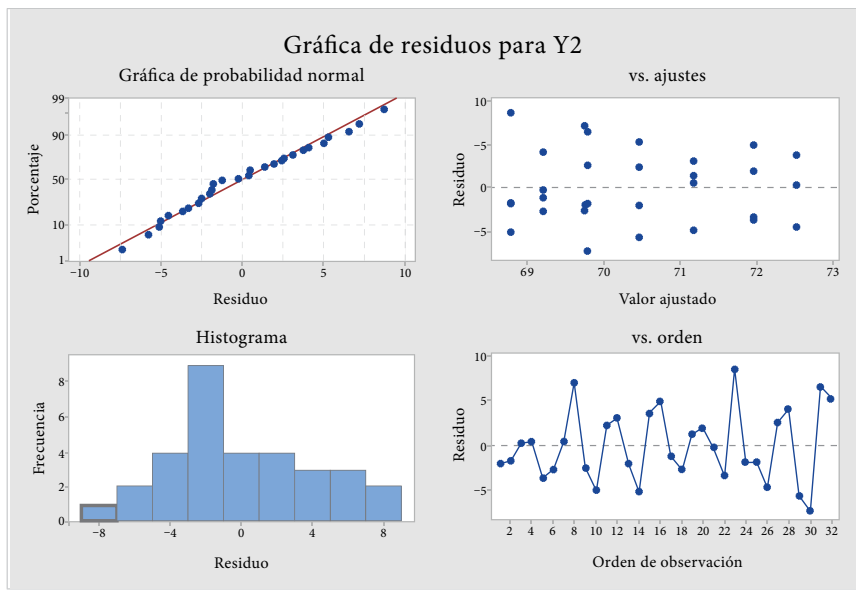

Figura 5. Independencia de residuales $Y_{2}$.

La Tabla 3 presenta el resultado del ANOVA. De igual manera, al considerar un nivel de significancia de 0.05 se puede notar que ninguno de los términos es significante, dado que en todos se tiene un valor $\mathrm{p}$ mayor que 0.05 .

TABLA 3

ANOVA PARA $Y_{2}$

\begin{tabular}{|l|r|r|r|r|c|}
\hline \multicolumn{1}{|c|}{ FUENTE } & GL & \multicolumn{1}{c|}{$\begin{array}{c}\text { SC } \\
\text { AJUST. }\end{array}$} & $\begin{array}{c}\text { MC } \\
\text { AJUST. }\end{array}$ & $\begin{array}{c}\text { VALOR } \\
\text { F }\end{array}$ & $\begin{array}{c}\text { VALOR } \\
\text { p }\end{array}$ \\
\hline Modelo & 7 & 50.235 & 7.1765 & 0.34 & 0.928 \\
\hline Lineal & 3 & 22.299 & 7.4331 & 0.35 & 0.788 \\
\hline$X_{1}$ & 1 & 1.363 & 1.3629 & 0.06 & 0.802 \\
\hline$X_{2}$ & 1 & 9.915 & 9.9147 & 0.47 & 0.500 \\
\hline$X_{3}$ & 1 & 11.022 & 11.0219 & 0.52 & 0.477 \\
\hline Interacciones de 2 términos & 3 & 18.808 & 6.2695 & 0.30 & 0.828 \\
\hline$X_{1} \times X_{2}$ & 1 & 0.213 & 0.2133 & 0.01 & 0.921 \\
\hline$X_{1} \times X_{3}$ & 1 & 4.646 & 4.6460 & 0.22 & 0.644 \\
\hline$X_{2} \times X_{3}$ & 1 & 13.949 & 13.9491 & 0.66 & 0.425 \\
\hline Interacciones de 3 términos & 1 & 9.128 & 9.1277 & 0.43 & 0.518 \\
\hline$X_{1} \times X_{2} \times X_{3}$ & 1 & 9.128 & 9.1277 & 0.43 & 0.518 \\
\hline Error & 24 & 507.691 & 21.1538 & & \\
\hline Total & 31 & 557.926 & & & \\
\hline
\end{tabular}


Con el objetivo de establecer una relación cuantitativa entre las variables y de esta manera llevar a cabo predicciones ajustadas, a continuación se muestran las ecuaciones de regresión para las variables $Y_{1}$ y $Y_{2}$ respectivamente, donde se expone como el comportamiento de la variable dependiente es dictado por una o más variables independientes.

$$
\begin{array}{r}
Y_{1}=104.867+1.419 X_{1}+0.107 X_{2}-0.688 X_{3}- \\
\left(0.325 X_{1} \times X_{2}\right)+\left(0.533 X_{1} \times X_{3}\right)-\left(0.104 X_{2} \times X_{3}\right)+ \\
\left(0.033 X_{1} \times X_{2} \times X_{3}\right) \\
\\
\\
Y_{2}=70.451+0.206 X_{1}-0.557 X_{2}+0.587 X_{3}+ \\
\left(0.082 X_{1} \times X_{2}\right)-\left(0.381 X_{1} \times X_{3}\right)-\left(0.660 X_{2} \times X_{3}\right)- \\
\left(0.534 X_{2} \times X_{3}\right)
\end{array}
$$

\section{B. Optimización de múltiples variables de respuesta}

A continuación, se muestra el proceso de optimización mediante la optimización restringida. En general, para este tipo de problemas con múltiples variables de respuesta son utilizados algoritmos de programación no lineal, estrategia que radica en definir la función de una respuesta como función objetivo a optimizar y definir valores objetivos o límites para las demás funciones. En este caso se cuenta con el siguiente planteamiento:

$$
\begin{gathered}
\text { máx } Y_{1} \\
\text { s. } a . \\
71 \leq Y_{2} \leq 75 \\
-1 \leq X_{1} \leq 1 \\
-1 \leq X_{2} \leq 1 \\
-1 \leq X_{3} \leq 1
\end{gathered}
$$

En este problema se encuentra como función objetivo el maximizar la función $Y_{1}$ sujeta a $Y_{2}$ con límites entre 71 y 75. Para finalizar, se definieron restricciones para $X_{1}, X_{2}$ y $X_{3}$ y como límites los valores mínimos y máximos de experimentación para cada factor que son -1 y 1 .

El problema de optimización puede ser solucionado a través del uso de diversos algoritmos de programación no lineal. Para este caso se recomienda el uso de un software especializado como RStudio, que fue elegido para este caso de estudio.

En primer lugar, se programó un algoritmo para poder encontrar los resultados óptimos (Tabla 4). El paquete seleccionado fue Rsolnp, el cual está basado en el método de multiplicadores de Langrage aumentado [22].
TABLA 4

Algoritmo de Optimización RsolnP

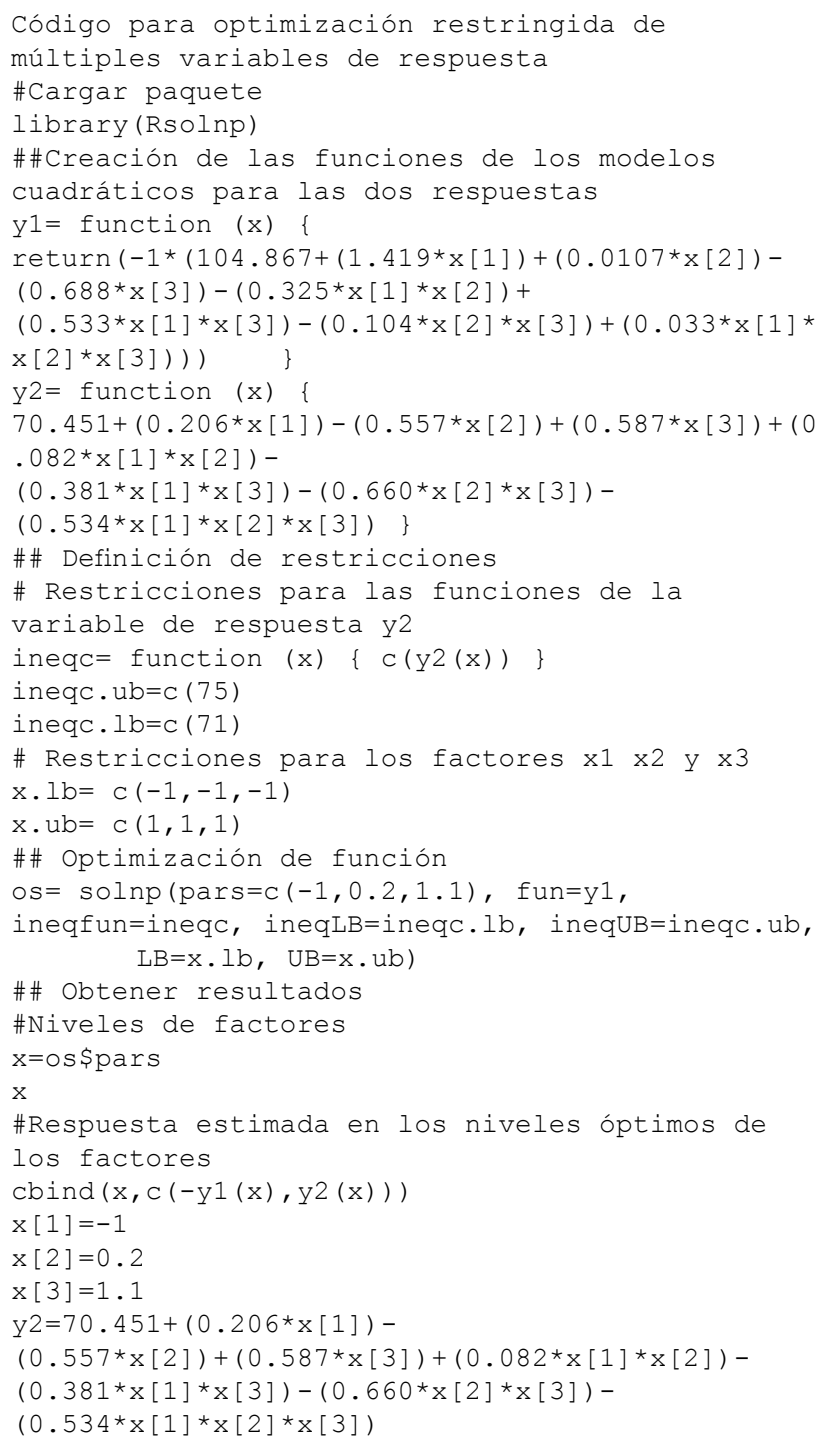

Como resultado, se obtuvieron los valores siguientes: $X_{1}=-1.0, X_{2}=0.2, X_{3}=1.1, Y_{1}=102.14190$ y $Y_{2}=$ 71.15428, cuya interpretación indica un nivel de optimización aceptable, debido a que los objetivos previamente planteados fueron alcanzados, principalmente con base en la maximización de la función $Y_{1}$. Por otra parte, el valor de la variable $Y_{2}$ se encuentra dentro de los límites establecidos.

Una vez resuelta la optimización mediante el software Rstudio, se llevó a cabo el cálculo de las funciones de deseabilidad y gráficos de contorno, los cuales se ejecutaron en el software Minitab ${ }^{\circledR}$, con los que se alcanzaron los resultados siguientes. 
En el gráfico de contornos que se muestra en la Figura 6 fue obtenido mediante el uso de Minitab ${ }^{\circledR}$, se puede visualizar una región de color blanco, la cual indica una región factible y cumple con todas las restricciones orientadas de manera gráfica, donde cualquier valor para $X_{1}$ y $X_{2}$ se considera una respuesta óptima.

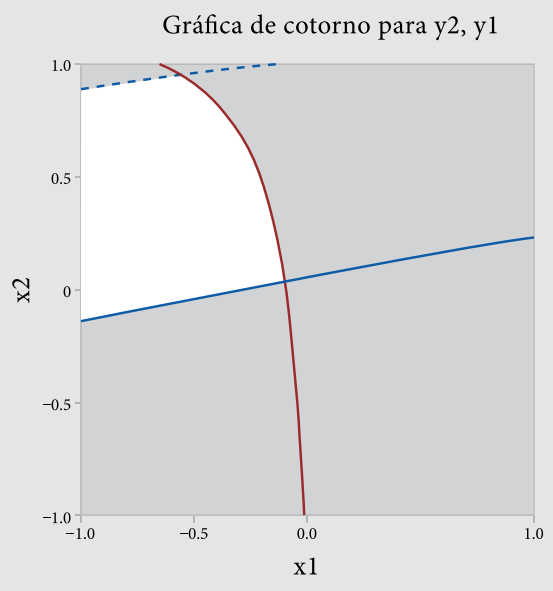

Figura 6. Gráfico de contornos superpuesto.

Las funciones de deseabilidad que se muestra en la Figura 7 fueron obtenidas también mediante el uso de Minitab $^{\circledR}$. Se puede notar que para las diferentes variables se obtuvo lo siguiente: $X_{1}=-1.0, X_{2}=0.3737, X_{3}=$ $1.0, Y_{1}=107.0909$ y $Y_{2}=72.9969$, lo cual indica una conveniente optimización, ya que fue posible alcanzar los objetivos, donde el valor de la función es el máximo posible $Y_{1}$ mientras que el valor de la función $Y_{2}$ se encuentra dentro de los límites señalados. De igual manera, en las variables se logró un efecto dentro de los límites asignados.

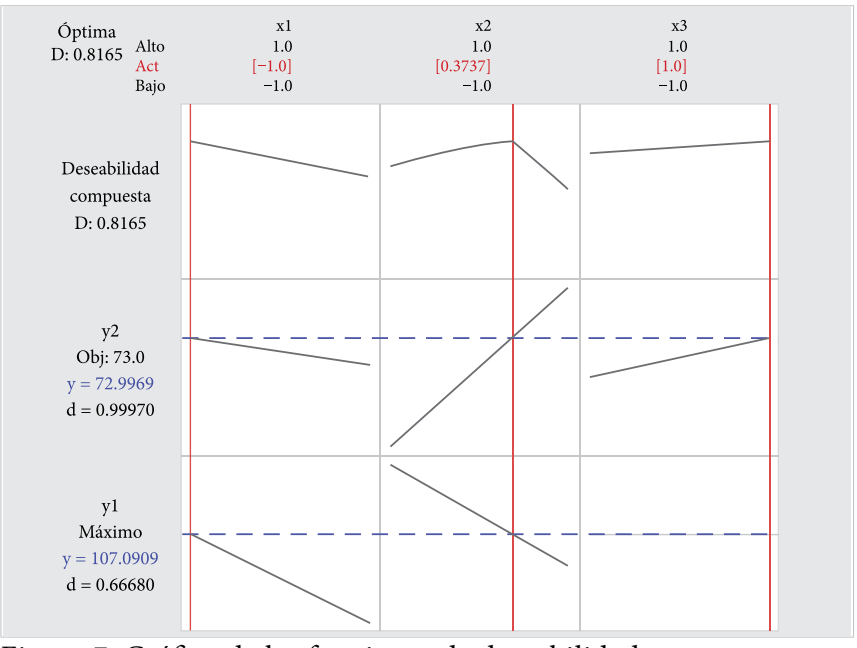

Figura 7. Gráfico de las funciones de deseabilidad.

\section{Comparación de resultados}

Al considerar los resultados obtenidos, tal como se observan en la Tabla 5, mediante la experimentación de las tres estrategias se puede afirmar que los tres métodos lograron los objetivos y restricciones establecidas. Por tanto, se concluye que cualquiera de los métodos es apto para optimizar ciertas variables.

TABLA 5

Comparación de Resultados

\begin{tabular}{|l|c|c|c|}
\hline & $\begin{array}{c}\text { PROGRAMACIÓN } \\
\text { NO LINEAL }\end{array}$ & $\begin{array}{c}\text { FUNCIONES DE } \\
\text { DESEABILIDAD }\end{array}$ & $\begin{array}{c}\text { GRÁfICO DE SUPER } \\
\text { CONTORNOS }\end{array}$ \\
\hline$Y_{1}$ & 102.14190 & 107.0909 & Véase la Figura 6 \\
\hline$Y_{2}$ & 71.15428 & 72.9969 & Véase la Figura 6 \\
\hline$X_{1}$ & -1.0 & -1.0 & -1.0 \\
\hline$X_{2}$ & 0.2 & 0.3737 & 0.2 \\
\hline$X_{3}$ & 1.1 & 1.0 & 1.0 \\
\hline
\end{tabular}

Se puede observar que el método de funciones de deseabilidad proporcionó un valor máximo mayor para $Y_{1}$, estableciéndose así como el preferible para la realización de este tipo de situaciones, ya que también se observa que $Y_{2}$ resultó dentro de las restricciones donde debía ser mayor a 71 y menor a 75 .

Por supuesto, el método gráfico no tiene la ventaja de proporcionar valores específicos de los factores que optimizan las variables de respuesta, sino que presentan solo una región óptima que el analista podría considerar para elegir un conjunto de puntos óptimos. Por otra parte, los métodos de funciones de deseabilidad y programación no lineal tienen la gran ventaja de presentar valores específicos de los diferentes factores para optimizar las múltiples respuestas. Definitivamente, un siguiente paso consistiría en validar los valores óptimos encontrados con la intención de comprobar el logro de las variables de respuesta óptimas.

\section{CONCLUSIONES}

El diseño de experimentos es conocido como una excelente herramienta apta para lograr la optimización de ciertas respuestas mediante la combinación de distintas variables. En este artículo se presentan distintas estrategias que pueden ser aplicadas para lograr este tipo de cuestiones.

Entre ellas se encuentra el método gráfico, que es factible porque se utilizaron dos factores, dejando fijo el tercero. Las funciones de deseabilidad consisten prácticamente 
en un método donde es necesario definir una función que mide la deseabilidad con el fin de transformar un problema de optimización con múltiples variables de respuesta a un problema de optimización univariado. Por último, se menciona la programación no lineal, que es el conjunto de métodos utilizados para optimizar una función objetivo, sujeta a ciertas restricciones en las que una o más de las variables es no lineal.

El objetivo de la aplicación de los métodos fue maximizar la función objetivo $Y_{1}$, respetando los límites establecidos para las distintas variables. El primero consiste en la aplicación de un algoritmo por medio del software RStudio, el cual se presenta como un paquete capaz de proveer un entorno donde es fácil de realizar un análisis de los datos obtenidos. La relevancia de la investigación reside en la capacidad para aplicar una estrategia experimental que permite obtener condiciones operativas deseables, frente a variables con cierto grado de correlación. En los tres métodos presentados, los resultados de optimización que se generan son similares.

Definitivamente, la gran mayoría de los productos cuentan con más de una característica crítica, las cuales por lo regular se encuentran correlacionadas, lo que genera que en algunas ocasiones los cambios en los niveles del diseño pueden mejorar una de las características mientras afectan adversamente una o más de las otras.

La situación planteada en el artículo es básicamente teórica, pero los métodos aquí ilustrados pueden ser considerados para la aplicación posterior dentro de la industria, ya que a partir de esta experiencia se pueden llegar a mejorar las condiciones y hacer cambios con el fin de prevenir problemas y reducir la variación. En ese sentido, la planeación experimental es un método importante dentro del repertorio de la ingeniería industrial. Por tal situación, se recomienda que como trabajo futuro se realice cierta comparación de estrategias en una aplicación de la rama industrial.

\section{REFERENCIAS}

[1] G. Ramón, Diseños experimentales. Apuntes de clase del curso Seminario Investigativo VI, Antioquía: Universidad de Antioquía, 2015, pp. 1-38.

[2] H. Gutiérrez-Pulido y R. Vara-Salazar, Análisis y diseño de experimentos, 2. ${ }^{\mathrm{a}}$ ed. México: McGraw-Hill/Interamericana Editores, 2008.
[3] M. J. Cervantes y T. F. Engstrom, "Factorial Design Applied to CFD”, J. Fluids Eng., vol. 126, no. 5, sept. 2004, doi: 10.1115/1.1792277.

[4] L. V. Bacio, "Optimización Multi-Objetivo en el Problema de Metodología de Superficie Multi-Respuesta", tesis de maestría, Centro de Investigación en Matemáticas, A. C., Guanajuato, Gto., México, 2007. [En línea]. Disponible: https:/cimat.repositorioinstitucional.mx/ jspui/bitstream/1008/69/2/TE\%20221.pdf

[5] J. J. Pignatiello Jr., "Strategies for Robust Multiresponse Quality Engineering", IIE Trans, vol. 25, no. 3, pp. 5-15, 1993, doi: 10.1080/07408179308964286.

[6] T. K. Trinh y L. S. Kang, "Response surface methodological approach to optimize the coagulation-flocculation process in drinking water treatment", Chem Eng Res Des, vol. 89, no. 7, pp. 1126-1135, 2011, doi: 10.1016/j. cherd.2010.12.004.

[7] Z. He, J. Wang, J. Oh y S. H. Park, "Robust optimization for multiple responses using response surface methodology", Appl. Stoch. Models Bus. Ind., vol. 26, no. 2, pp. 157-171, mar. 2010, doi: 10.1002/asmb.788.

[8] S. Shamsudeen y J. E. R. Dhas, "Optimization of Multiple Performance Characteristics of Friction Stir Welded Joint with Grey Relational Analysis", Mater. Res., vol. 21, no. 6, Nov. 2018, doi: 10.1590/1980-5373-mr-2017-1050.

[9] S. Jambhale, S. Kumar y S. Kumar, "Multi-response optimization of friction stir spot welded joint with grey relational analysis", Mater. Today: Proc., vol. 27, pp. 19001908, 2020, doi: 10.1016/j.matpr.2020.03.830.

[10] D. Kilic, M. Ebegil, H. Bayrak, B. Ozkaya y B. Apaydin, "Optimization Of Multi Responses Using Data Envelopment Analysis: The Application in Food Industry", Gazi Univ. J. Sci., vol. 32, no. 3, pp. 1083-1090, sept. 2019, doi: 10.35378/gujs.394984.

[11] O. I. Awad et al., "Response surface methodology (RSM) based multi-objective optimization of fusel oil -gasoline blends at different water content in SI engine", Energy Convers. Manag., vol. 150, pp. 222-241, oct. 2017, doi: 10.1016/j.enconman.2017.07.047.

[12] M. Mia, "Multi-response optimization of end milling parameters under through-tool cryogenic cooling con- 
dition", Measurement, vol. 111, pp. 134-145, dic. 2017, doi: 10.1016/j.measurement.2017.07.033.

[13] P. Achuthamenon Sylajakumari, R. Ramakrishnasamy, G. Palaniappan y R. Murugan, "Multi-response Optimization of End Milling Parameters for Al-Zn-Mg/ $\mathrm{SiC}$ Co-continuous Composite Using Response Surface Methodology", Materials Science, vol. 25, no. 4, pp. 471477, jun. 2019, doi: 10.5755/j01.ms.25.4.21000.

[14] R. De la Vara-Salazar y J. Domínguez-Domínguez, "Métodos de superficie multirespuesta: un estudio comparativo", Rev. Mat., vol. 9, no. 1, pp. 47-65, 2002, doi: 10.15517/rmta.v9i1.209.

[15] M. R. Hasniyati, H. Zuhailawati y S. Ramakrishnan, “A statistical prediction of multiple responses using overlaid contour plot on hydroxyapatite coated magnesium via cold spray deposition", Procedia Manuf., vol. 19, pp. 181-188, 2016, doi: 10.1016/j.proche.2016.03.091.

[16] S. I. Rudnykh y V. I. López-Ríos, “Elección de la función de deseabilidad para diseños óptimos bajo restricciones”, Revista EIA, vol. 15, no. 30, pp. 13-24, 2018, doi: 10.24050/reia.v15i30.903.

[17] X. Chen y J. Chen, "Optimization of the Impeller Geometry for an Automotive Torque Converter Using
Response Surface Methodology and Desirability Function", Open J. Appl. Sci., vol. 10, no. 07, 2020, doi: 10.4236/ojapps.2020.107032.

[18] E. Hazir, E. S. Erdinler y K. H. Koc, "Optimization of CNC cutting parameters using design of experiment (DOE) and desirability function", J. For. Res., vol. 29, pp. 1423-1434, 2018, doi: 10.1007/s11676-017-0555-8.

[19] R. Świercz, D. Oniszczuk-Świercz y T. Chmielewski, "Multi-response optimization of electrical discharge machining using the desirability function", Micromachines, vol. 10, no. 1, 2019, doi: 10.3390/mi10010072.

[20] R. Cantú-Cuéllar, "Programación no lineal”, tesis de maestría, Universidad Autónoma de Nuevo León, San Nicolás de los Garza, N. L., México, 1996. [En línea]. Disponible: http://cdigital.dgb.uanl.mx/te/1080072405. pdf

[21] E. Del Castillo, Process Optimization: a Statistical Approach. Boston, MA: Springer Science+Business Media, LLC, 2007.

[22] S. Kawakita et al., "Evaluation of non-linear wheat development models and optimization methods for their parameter determination", J. Agric. Meteorol., vol. 75, no. 2, 2019, doi: 10.2480/agrmet.D-18-00034. 